\title{
Neue Daten zu Imatinib und Everolimus
}

— Auf der 47. Jahrestagung der American Society of Clinical Oncology (ASCO) in Chicago, USA, wurden neue Studienergebnisse zur adjuvanten Imatinib-Therapie (Glivec ${ }^{\circledR}$ ) bei gastrointestinalen Stromatumoren (GIST) und zu Everolimus (Afinitor ${ }^{\circledR}$ ) beim fortgeschrittenen Nierenzellkarzinom (RCC) vorgestellt. Hierbei standen die Ergebnisse der offenen, multizentrischen, prospektiven, randomisierten GIST-Studie „SSG XVIII/AIOA“, an der insgesamt 397 auswertbare Patienten teilnahmen, im Fokus des Interesses. Der Vergleich einer 36-monatigen mit einer 12-monatigen adjuvanten Imatinib-Behandlung bei Hochrisikopatienten ergab: Fünf Jahre nach Therapiebeginn blieben $65,6 \%$ der länger behandelten Patienten rezidivfrei (vs. 47,9\%; HR: 0,46; $\mathrm{p}<0,0001)$. Zu diesem Zeitpunkt lebten noch $92 \%$ der GIST-Patienten mit 36-monatiger Therapie (vs. 81,7\%; HR: 0,45; $p=0,019$ ). Damit wurde in dieser Phase-III-Studie erstmals ein Überlebensvorteil durch eine längere adjuvante Behandlungsdauer mit Imatinib bei Hochrisiko-Patienten nachgewiesen. Imatinib

\section{Zweitlinientherapie beim Prostatakarzinom: Cabazitaxel überwindet Resistenzen}

— Beim metastasierten hormonrefraktären Prostatakarzinom (HRPC) ist die Kombination aus Docetaxel und Prednisolon die Therapie der ersten Wahl. Ein Problem der Behandlung sei jedoch die sich entwickelnde Taxan-Resistenz, erklärte Dr. Bernhard Heinrich aus Augsburg: „Die Erkrankung progrediert unter Docetaxel bei $95 \%$ der Patienten innerhalb von 18 Monaten." Ursache der Resistenz ist die Überexpression des Multidrug-Resistance-1Gens (MDR-1), welches das Membranprotein P-Glykoprotein (PGp) kodiert. PGp transportiert die Taxane aus der Zelle und verhindert so deren antiproliferative Wirkung. Das neue Taxan Cabazitaxel (Jevtana ${ }^{\oplus}$ ) überwindet diese Resistenz. Es stabilisiert wie andere Taxane die Mikrotubuli der Zelle, unterbricht den Zellteilungszyklus in der G2M-Phase und hemmt so die Proliferation der Tumorzelle. Durch eine Modifikation am Taxanring hat die Substanz jedoch eine geringere Affinität zu PGp als Docetaxel und wird seltener aus der Zelle transportiert. Cabazitaxel ist seit April 2011 in Eur- wurde generell gut vertragen und es traten keine unerwarteten Nebenwirkungen auf. Die häufigsten Nebenwirkungen waren Anämie, periorbitale Ödeme und erhöhte Lactatdehydrogenase-Werte.

Darüber hinaus wurden die Ergebnisse einer Interims-Analyse der prospektiven, nicht interventionellen "CHANGEB-Studie" präsentiert: Hier wurde im Praxisalltag beim fortgeschrittenen RCC unter Everolimus nach dem ersten Tyrosinkinaseinhibitor eine mediane Zeit bis zum Progress von 9,7 Monaten beobachtet. Somit ist die mediane Zeit bis zum Fortschreiten der Erkrankung nahezu doppelt so lange wie in der zulassungsrelevanten Phase-III-Studie RECORD ${ }^{\mathrm{C}}$-1 (4,9 Monate; $p<0,001)$. Nach Bergman et al. sprechen die Daten dieser Zwischenauswertung für den Einsatz von Everolimus bei Patienten mit fortgeschrittenem RCC nach Progress unter der ersten gegen VEGF-gerichteten Therapie.

Nach Informationen von

Novartis, Nürnberg kurz gemeldet

\section{High-Level-Desinfektion von Ultraschallsonden}

Das Unternehmen Hydes bietet speziell zur Desinfektion von Ultraschallsonden die High-Level-Desinfektion mit Trophon EPR ${ }^{\mathrm{TM}}$ an. Funktion und Wirksamkeit von Trophon EPR ${ }^{\mathrm{TM}}$ übertrifft alle vorgeschriebenen mikrobiologischen EN-Standards. Hersteller ist die australische Nanosonics Ltd. beziehungsweise die Nanosonics Europe $\mathrm{GmbH}$ mit Sitz in Hamburg.

Vorteile in der Praxis sind vor allem kurze Durchlaufzeiten, hervorragende Materialverträglichkeit und der Verzicht auf schädliche Chemikalien. Abgetötet werden die Sporen durch einen hochkonzentrierten, ultrafeinen Nebel, den sogenannten NanoNebulant $^{\mathrm{TM}}$. Eine High-Level-Desinfektion wird so in weniger als sieben Minuten erreicht: Die Ultraschallsonde ist sofort einsatzbereit, sie muss nicht mehr abgespült oder getrocknet werden.

Das Gerät unterstützt somit ideal die Prävention nosokomialer Infektionen und hilft so, die jährlich steigende Zahl infizierter Patienten durch kontaminierte Behandlungsgeräte zu bekämpfen.

Nach Information von HyDes, Altenkirchen

\section{Lösungen für Blasenprobleme}

Für jedes Blasenproblem gibt es eine passende Lösung. Bei einer akuten Blasenentzündung beispielsweise hat sich der Trockenextrakt der Bärentraube (Cystinol ${ }^{\circledR}$ akut Dragees) bewährt. Der Inhaltsstoff Arbutin wirkt antibakteriell und entzündungshemmend.

Bei einer rezidivierenden Blasenentzündung kann eine Kur mit echtem Goldrutenkraut (Cystinol long ${ }^{\circledR}$ Kapseln) helfen. Die durchspülende Wirkung schwemmt unerwünschte Erreger aus der Blase, und die entzündungshemmenden Eigenschaften heilen die angegriffene Blasenschleimhaut.

Bei einer Reizblase bietet sich eine Kombination aus den Extrakten der Bärentraube und der echten Goldrute (Cystinol ${ }^{\circledR} \mathrm{N}$ Lösung) an. Echte Goldrute beruhigt die entzündete Blasenschleimhaut und hat einen durchspülenden und krampflösenden Effekt.

Fachpresse-Workshop "2. Expertise Prostata“, Eltville am Rhein, 29. Juni 2011, Veranstalter: Sanofi-Aventis, Frankfurt
Nach Informationen von Schaper \& Brümmer, Salzgitter-Ringelheim 